\title{
Chloroquine and Hydroxychloroquine: how about switching?
}

\author{
Thiago Santana Novotny ${ }^{1,2 *}$, Mychelle Alves Monteiro ${ }^{1}$, Patrícia Condé de Lima ${ }^{1}$, Soraya Mendonça de Ochs ${ }^{1}$, Fernando Antonio Simas Vaz ${ }^{2}$, \\ Flávia Ferreira de Carvalho Marques ${ }^{2}$ \\ ${ }^{1}$ National Institute for Quality Control in Health, Oswaldo Cruz Foundation, 21040-900, Rio de Janeiro, RJ, Brazil. \\ ${ }^{2}$ Department of Analytical Chemistry - Fundamental and Applied Analytical Chemistry Laboratory, Institute of Chemistry, Fluminense Federal \\ University, 24020-141, Niterói, RJ, Brazil.
}

Corresponding Author: Thiago Santana Novotny, Instituto Nacional de Controle de Qualidade em Saúde (INCQS) - Fundação Oswaldo Cruz (Fiocruz), Avenida Brazil.

Received date: February 24, 2021; Accepted date: June 21, 2021; Published date: December 03, 2021

Citation: Thiago S. Novotny, Mychelle A. Monteiro, Patrícia C. Lima, Soraya M. Ochs, Fernando Antonio S. Vaz, Flávia F. C. Marques (2021) Chloroquine and Hydroxychloroquine: how about switching? J. Pharmaceutics and Pharmacology Research. 4(4); DOI: 10.31579/2693-7247/034

Copyright: ( $)$ 2021, Thiago Santana Novotny, This is an open access article distributed under the Creative Commons Attribution License, which permits unrestricted use, distribution, and reproduction in any medium, provided the original work is properly cited.

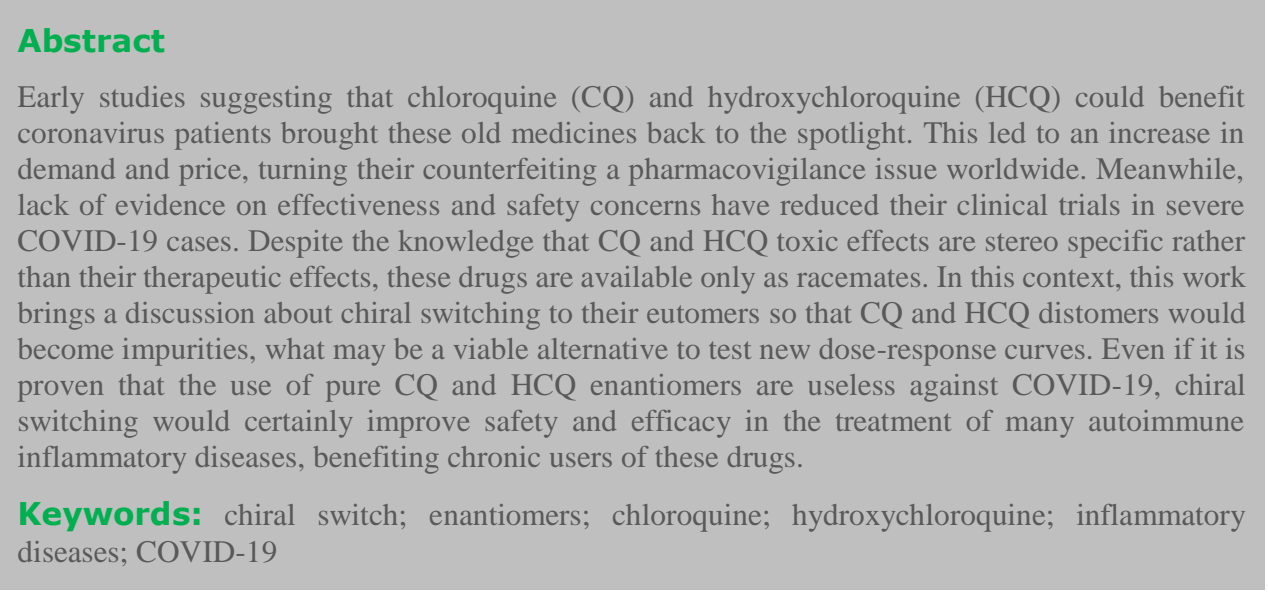

Chemical compounds: chloroquine (PubChem CID: 2719); hydroxychloroquine (PubChem CID: 3652); quinine (PubChem CID: 3034034); desethyl chloroquine (PubChem CID: 95478); bis-desethyl chloroquine (PubChem CID: 122672); mono desethyl hydroxychloroquine (PubChem CID: 71826).

\section{Introduction: Why is this fuss all about?}

In late 2019, a group of patients with a severe acute respiratory syndrome (SARS) of unknown cause appeared in Wuhan, Hubei province, China (Timeline of WHO's Response to COVID-19, n.d.). Since then, outbreaks of this syndrome had quickly spread across Chinese territory, resulting in thousands of confirmed cases. The Chinese Center for Disease Control and Prevention organized an intensive outbreak investigation program and attributed the etiology of this disease to a new virus belonging to the coronavirus (CoV) family. Initially, the new virus was called 2019-nCoV, and on February $11^{\text {th }}$ of 2020 the World Health Organization (WHO) announced that the disease caused by the new CoV should be called "COVID-19", which stands for "coronavirus disease 2019". Subsequently, experts from the International Committee on Taxonomy of
Viruses (ICTV) renamed the newly discovered CoV to SARS-CoV-2 due to its similarity to the coronavirus responsible for the first epidemic of SARS (SARS-CoV) in $2002[12,23]$. Because of its alarming speed of transmission COVID-19 advanced worldwide and reached all continents, causing the WHO to declare a pandemic state on March $11^{\text {th }}$ of the same year (Coronavirus Disease (COVID-19), n.d.).

By February of 2021, almost a year since the pandemic was declared, there were more than 106 million confirmed cases, and more than 2, 3 million deaths throughout 222 affected countries (Coronavirus Disease (COVID-19), n.d.). Although SARS-CoV-2 is not the most lethal among coronaviruses, its speed of propagation makes it one of the deadliest viruses known. Nonetheless, several vaccines have been developed in record time and promise to be effective against the disease. However, they are being used on an emergency basis pending regulatory approval for obtaining definitive registration in many countries. While mass vaccination does not start globally, the pandemic is advancing and many countries have resumed actions of social distance and restrictions on commercial activities (COVID-19 Vaccines, n.d.). Meanwhile, treatment for COVID-19 remains symptomatic and supportive. In a race against 
time, scientists are trying to repurpose the use of old medicines. It has been reported that most of COVID-19 patients received antiviral therapy in China. Current treatment options are mainly based on previous experience that have shown clinical benefits in treating influenza, Ebola, the Middle East Respiratory Syndrome (MERS), SARS, and other viral infections. Ribavirin, lopinavir, ritonavir, remdesevir, nelfinavir, arbidol have been tested but none of which proved to be effective against COVID19. In vitro data have shown that some anthelmintic, such as nitazoxanide, ivermectin and niclosamide, inhibit replication of a variety of viruses, leading them to be tested against COVID-19. However, in vivo results are poor and inconclusive [54, 70].

Huge inflammatory responses known as cytokine storms are one of the features of severe cases of COVID-19 and are often associated with increased mortality, so the use of systemic anti-inflammatory drugs is a potential therapeutic strategy. However, timely and appropriate use of corticosteroids, such as methylprednisolone, are recommended only to patients that are presenting ongoing deterioration in the oxygenation index, or rapid progression of radiological findings, or excessive activation of immune responses. Together with immunosuppressive agents, broad-spectrum antibiotics, such as carrimycin and azithromycin, have also been prescribed as adjunct therapies to prevent bacterial secondary infection. [15, 23, 54, 70 and 79].

Some antimalarial drugs have shown to have immunomodulatory/antiinflammatory properties and antiviral activity as well. Since the late 1960s it is known that chloroquine (CQ) can inhibit the growth and spread of different viruses, (including the SARS-CoV) in vitro, but subsequent in vivo experiments were controversial (Favalli et al., 2020). Early studies in China and France claiming the use of CQ and its derivative hydroxychloroquine (HCQ) could be beneficial to coronavirus patients brought these drugs back to the spotlight. The spread of these news and the adoption of CQ and HCQ in public policies to combat COVID-19 by some of the largest world economies, such as the United States and Brazil, have turned these drugs by far the most popular proposed for treatment and prophylaxis, appearing in several clinical studies registered on the United States National Institutes of Health (NIH) website [16, 28, 40, 47, 56, 64 and 67].

These apparently good news led to an increase in demand and selfmedication with CQ and HCQ, raising concerns about possible retinopathy and serious cardiac impairment by these drugs. These concerns were confirmed in some of the clinical trials performed. In addition to an increased risk of cardiac complications, CQ and HCQ do not resulted in the reduction of mortality of hospitalized COVID-19 patients, when compared with standard of care, what has prompted WHO to discontinue clinical trials using these drugs in critically ill patients. However, clinical trials to evaluate the efficacy of CQ and HCQ in pre or post-exposure prophylaxis are still ongoing. Although there is already evidence that HCQ has no effect in altering the course of the disease in individuals without comorbidities, it is possible that this drug is more beneficial for patients who are at higher risk groups. More clinical trials need to be conducted in this regard [46, 60 and 64].

Furthermore, due to its widespread use as an antimalarial, CQ has been one of the most counterfeit medicines for many years. CQ and HCQ are also widely used in the treatment of autoimmune inflammatory diseases like lupus erythematous and rheumatoid arthritis due to their off-label immunomodulatory activity. The increase in demand due to its popularization in the treatment of COVID-19 and the lockdown imposed on the two largest producers of active pharmaceutical ingredients, China and India, caused a shortage in the market and the prices to skyrocket. This, in addition to putting at risk the continuity of treatment of the patients who make chronic use of these drugs, ended up triggering an increase in CQ counterfeiting around the world, turning it into a major pharmacovigilance issue [5].

However, CQ and HCQ are currently marketed as racemates, a mixture of equal quantities of two enantiomers. It is known that the enantiomers in a drug available as racemic mixture may have distinct pharmacological and/or toxicological properties. Chiral switch is the substitution in the marketplace of a racemic drug for its single-enantiomer version [29]. Chiral switching CQ and HCQ to their eutomers, the enantiomers that cause the desired therapeutic responses, may be an alternative to reduce their toxic adverse effects, allowing a safer administration of higher doses and a better evaluation of the effectiveness against COVID-19 over a wider dose-response range. In addition, the marketing of the pure eutomer could improve the quality of life for those who continuously take these drugs, and also turn the enantiomeric purity assessment and useful indicator for counterfeit and substandard medicines identification [2, 37]. Therefore, these molecules still have properties that should be properly exploited to really exhaust all possibilities against diseases.

\section{SARS-CoVs mechanism of infection}

With a diameter of approximately 60 to $140 \mathrm{~nm}$, round or elliptical shaped and often pleomorphic, coronaviruses (CoVs) are RNA viruses which strand of genetic material is the longest known. According to their genetic characteristics, coronaviruses are classified into four genera: a-CoV, b$\mathrm{Cov}, \mathrm{g}-\mathrm{CoV}$ and d-Cov. Genetic sequencing of the coronavirus isolated from patients who contracted COVID-19 revealed that SARS-CoV-2 belongs to the b-CoV genus. [12, 23 and 79]. CoVs are given this name because of their appearance as a crown (coronam, crown in Latin) when viewed under the electron microscope due to the presence of spicules of glycoproteins around their envelope that serve as a docking point at the host cell.

The SARS-CoVs glycoprotein spikes are comprised of two subunits, S1 and S2. During the infection of the human cell, the $S 1$ subunit binds to an angiotensin-converting enzyme type 2 (ACE-2) like receptor located in the membrane of the host cell. The virus can then enter the cell either through endocytosis or can fuse its membrane to the cell's membrane and release its genome and protein content directly into the cytoplasm (Figure $1)$. 


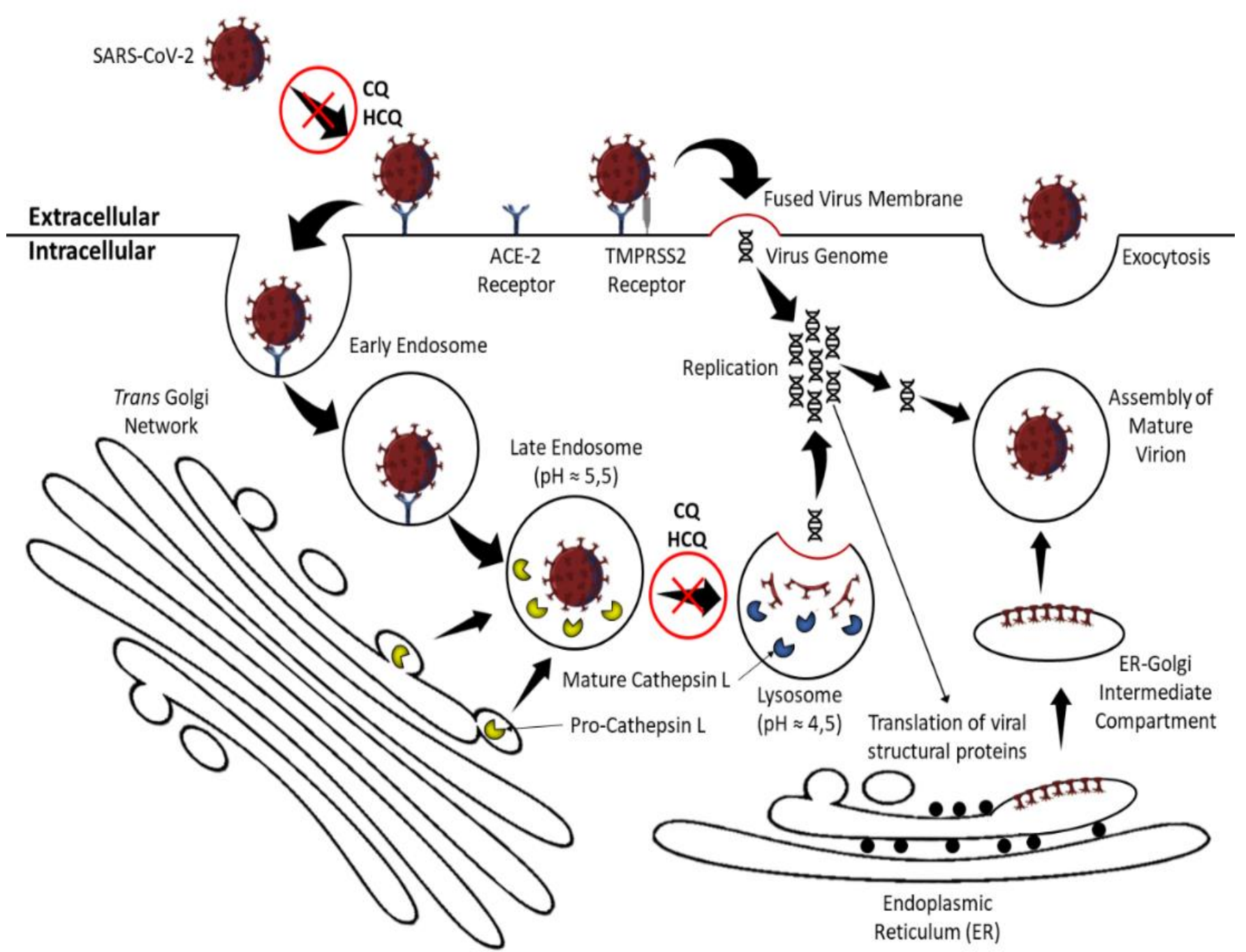

Figure 1. Mechanism of SARS-CoV-2 entry into the host cell and $Q C$ and $H C Q$ action points. CQ and HCQ can interfere with the binding of the virus to ACE-2 receptors and prevent the endosomes from maturing to the late form. A similar process is involved

Either way the ACE-2 bound spike must be cleaved by specific proteases to initiate the virus replication process. At the cell surface the spike is cleaved mainly by the transmembrane protease serine-2 (TMPRSS2), ensuring virus and host-cell membrane fusion. In the endocytosis pathway the spike is cleaved by cathepsin L within the endosome, provoking the fusion between the viral membrane and the endocytic membrane. These two mechanisms ultimately result in cell infection [12, 23]. (Hoffmann, Kleine-Weber, \& Pöhlmann, 2020; Hoffmann, Kleine-Weber, Schroeder, et al., 2020; Singh et al., 2020).

\section{COVID-19: who are the players?}

\subsection{The role of the angiotensin converting enzyme}

The angiotensin converting enzyme (ACE) is a transmembrane enzyme widely distributed in the body's tissues and is an essential component of the renin-angiotensin-aldosterone system (RAAS), mediating a number of systemic and local effects in blood pressure control, tissue perfusion and extracellular volume (Figure 2).

ACE is a dicarboxy-peptidase enzyme that acts removing the carboxyterminal dipeptide from the decapeptide angiotensin I (Ang I) resulting in the octapeptide angiotensin II (Ang II; Ang I-8). By binding to the angiotensin II (Ang II) type 1 receptors $\left(\mathrm{AT}_{1}\right)$ in the cardiovascular system, Ang II plays an important role in blood pressure homeostasis, acting on multiple organs through intense vasoconstriction, aldosterone release from the adrenal glands, pituitary secretion of vasopressin and reabsorption of sodium and water at the kidneys [22, 39 and 62]. 


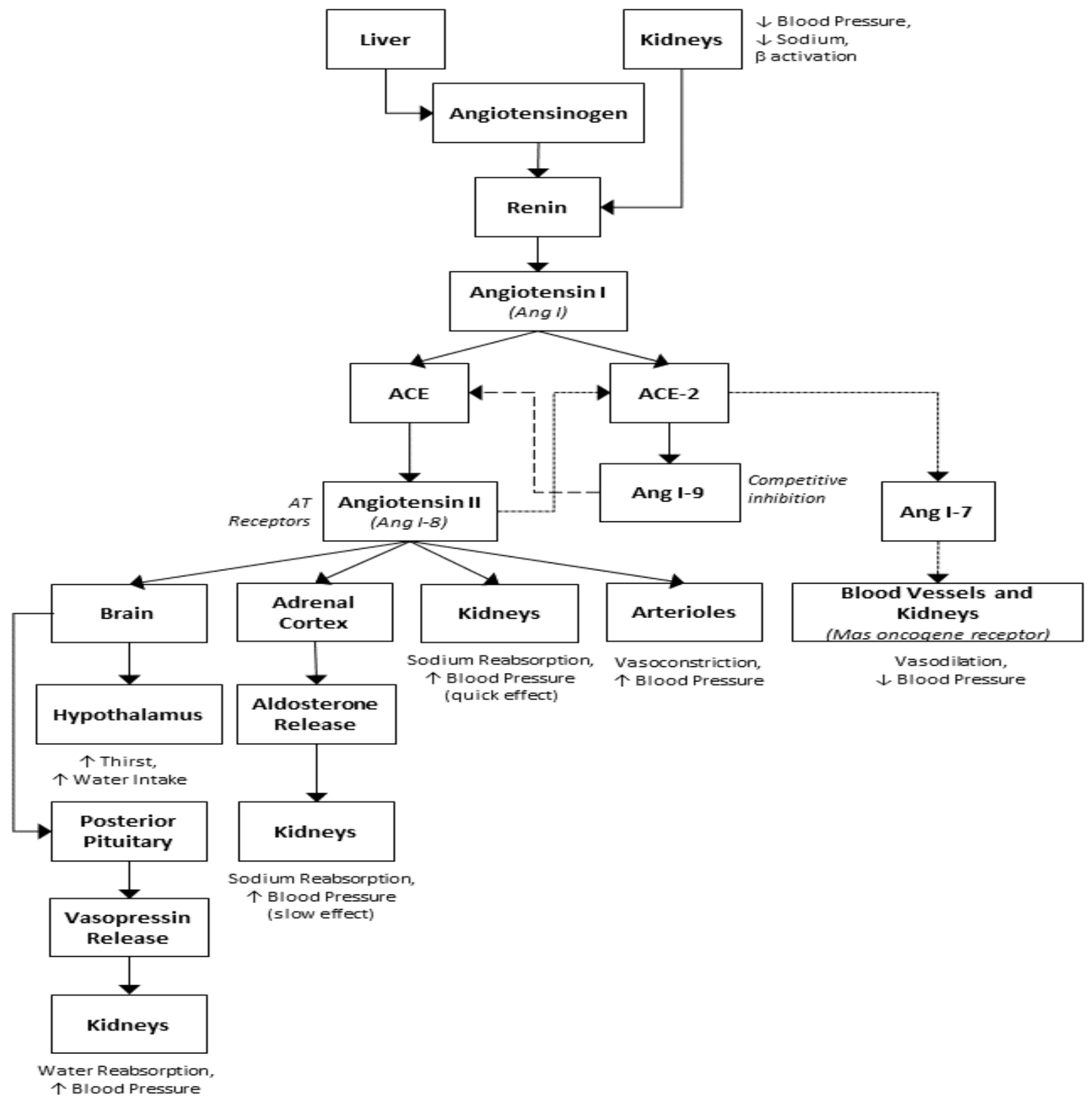

Figure 2. The Renin-Agiotensin System: Renin is activated in the juxtaglomerular cells of the kidneys in response to decreased blood pressure, betaactivation, or activation by macula densa cells in response to a decreased sodium load in the distal convo.

The ACE homologous enzyme, ACE-2, plays a fundamental physiological role in maintaining local homeostasis. Despite a more than $42 \%$ homology in the amino acid sequence of their catalytic sites, ACE and ACE-2 are biochemically and pharmacologically distinct. ACE-2 is a monocarboxypeptidase and, unlike ACE, it is only capable of hydrolyzing one amino acid at a time from the carboxy-terminal portion of its substrate. Thus ACE-2 converts the decapeptide Ang I into the nonapeptide Ang I-9 and converts the octapeptide Ang II to the heptapeptide Ang I-7. Although the function of Ang I-9 is not fully understood, it is suggested that it acts as an endogenous competitive inhibitor of ACE, since Ang I-9 is also a substrate for ACE. Ang I-7, in turn, produces a cardiovascular and renal vasodilator effect when it binds to the Mas oncogene receptor, which negatively regulates the effects of Ang II mediated by the $\mathrm{AT}_{1}$ receptor (Figure 2). This indicates the important protective physiological role played by ACE- 2 in counter to the effects of Ang II. [22, 30, 52 and 59].

ACE-2, is present in various organs and tissues, such as the bronchi, bronchioles and lungs, cardiovascular system (including the coronary heart vessels), renal tubular epithelium, esophagus, intestines, testes, endocrine pancreas, bile ducts, retina and brain. However, its distribution is not uniform meaning that some organs might be more prone to infection than others. This can explain the spread of the virus beyond the respiratory 
tract and the damage to certain organs found in the severe form of the disease [12, 23 and 59].

\subsection{The role of angiotensin II in the inflammatory process}

Ang II is also involved in the development of pro-inflammatory stimuli in different organs and tissues. Overexposure of cells to Ang II increases the expression of $\mathrm{AT}_{1}$ and $\mathrm{AT}_{2}$ receptors. The activation of $\mathrm{AT}_{1}$ receptors by Ang II, in addition to promoting the production of reactive oxygen species (ROS) that lead the cell to oxidative stress, increases the production of the lectin-type oxidized low-density lipoprotein receptor 1 (LOX-1). LOX-1 increases the expression of messenger RNA for the formation of more $\mathrm{AT}_{1}$ receptors and the intracellular production of proinflammatory signaling molecules such as, interleukin-6 (IL-6), interleukin-10 (IL-10), tumor necrosis factor a (TNF-a) and intercellular adhesion molecules, favoring the action of monocytes of the immune system. The activation of $\mathrm{AT}_{2}$ receptors, in turn, increases the intracellular production of pro-apoptotic molecules. Thus, the expression/activation of $\mathrm{AT}_{1}$ and $\mathrm{AT}_{2}$ receptors in response to Ang II and the inflammatory environment is a feedback process that amplifies the inflammatory response and cell death in a chained reaction [74]. Cellular infection by SARS-CoVs decreases the availability and expression of ACE-2, unbalancing the local renin-angiotensin system (RAS) and favoring overstimulation by Ang II, which further intensifies the inflammatory response [36].

\subsection{Other immunologic features}

In addition, the rapid viral replication in the host cell causes massive pyroptosis at the pulmonary epithelium and endothelium, resulting in acute injuries and an intense inflammatory process in the lung tissue, leading to an abrupt decrease in respiratory capacity, typical in severe SARS-CoVs infections [27]. Unlike apoptosis (where cell death occurs in a programmed and organized manner, shrinking gradually until it is finally phagocyted), pyroptosis is a process of rapid and highly inflammatory cell death (Figure 3 ). The cascade of biochemical reactions triggered by pyroptosis ends up in the formation of an inflammasome and rupture of the cell membrane in a desperate attempt by the host cell to prevent the pathogen from using the cellular machinery for its own replication (Yang, 2020). The rupture of infected cells produces viremia (the release of new viral particles in the bloodstream) and spreads the infection to other organs and tissues where ACE-2 receptors are available. The pyroptosis process also provokes the extravasation of immunomodulatory molecules in the bloodstream, triggering inflammatory responses and mobilizing immune system cells such as, macrophages and $\mathrm{T}$ helper lymphocytes and the consequent release of interferon $\mathrm{g}$ (IFN-g), which leads to the recruitment of more inflammatory cells, initiating an inflammatory chain reaction known as "cytokine storm". This exaggerated and out-of-control response of the innate immune system causes tissue damage and is considered to be the main cause of acute respiratory syndrome and multiple organ failure seen in severe cases of COVID-19 [15, 27 and 59]. The risk of developing a cytokine storm is particularly high in elderly patients, who are more likely to have pre-existing chronic inflammation and an enabling inflammatory environment. The suppression of the cytokine storm with immunomodulatory drugs is an important way to prevent further aggravation of COVID-19 patients. However, this approach must be started at the right time, because if administered too early the pharmacological suppression of the immune system will make it difficult to eliminate the virus in the body [55, 77, 78 and 79].

Infection of immune cells also contributes to the spread of the virus from the lungs to other organs via the lymphatic circulation, competing with viremia for the rapid spread of the disease in the body. A probable mechanism for the spread of the virus via the immune system, which may be related to the reduction of defense cells and evolution to the severe form of the disease, is a phenomenon known as antibody-dependent enhancement (ADE). Antibodies produced after infection by a first viral agent can cross-react with a second viral agent (which does not need to belong to the same species) and produce clusters of antigens with antibodies or with components of the complement system that will be internalized by phagocytic cells through its FcRg type receptors. Once internalized through this mechanism, the virus is not destroyed. On the contrary, the viral particle replicates and leaves the host cell to infect others, and by leaving the cell it still induces it to secrete proinflammatory cytokines. This phenomenon has been described in other infections by SARS, MERS and Dengue virus and may explain the geographical variability of severe cases of COVID-19. Particularly worrying in the case of COVID-19 is that this infection mechanism is independent of ACE-2 receptors, and that there are other types of nonimmune cells that also have FcRg receptors. Interestingly, the lower the antibody titer remaining from the primary infection, the greater the potentiation of the secondary infection. Other types of coronaviruses can infect humans, causing mild flu or even no noticeable symptoms. Although these infections are not sufficient to cause seroconversion and produce a lasting immune response, during the post-convalescence period, when the antibody titer produced is low, is when there is the greatest risk for potentiation of the secondary infection dependent on antibodies produced in the primary one. This feature of the immune system in response to some viral infections imposes an additional challenge to produce a vaccine against SARS-CoV-2, where artificial immunization could actually increase the risk of developing the severe form of the disease [1, 49 and 72].

It is already known that the serum conversion caused by SARS-CoV-2 is not long-lasting and that IgG titers decline considerably after a few weeks after the period of convalescence. The ADE phenomenon has already been seen in other coronavirus infections. The occurrence and contribution of ADE to the evolution of severe cases of COVID-19 cannot be ruled out [42]. Therefore, immunomodulatory drugs could be useful in the treatment of artificially induced ADE and in preventing its natural occurrence as well. 


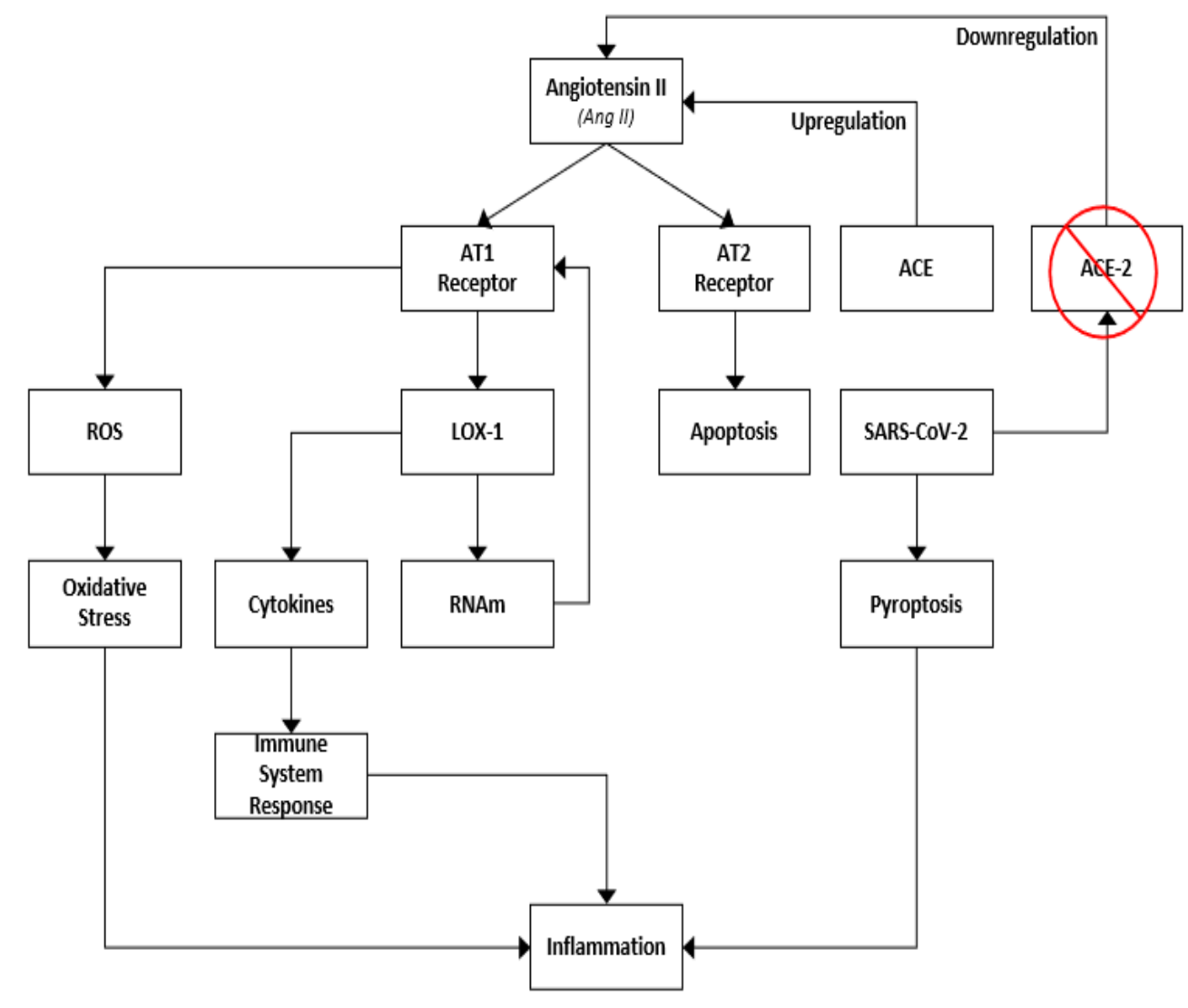

Figure 3. Role of the renin-angiotensin-aldosterone system in inflammatory processes triggered by the action of angiotensin II (Ang II) on AT receptors. Infection of the cell by SARS-CoV-2 aggravates inflammation by destroying ACE-2 receptors that counteract the effects of Ang II and by triggering pyroptosis in the infected cell.

\section{CQ and HCQ back to the spotlight}

\subsection{Immunomodulatory Effects}

CQ and HCQ were originally developed as anti-malarial drugs, but their accumulation in lymphocytes and macrophages results in antiinflammatory properties that has led them to an off label use as part of the standard strategy for the treatment of patients suffering from autoimmune diseases such as, rheumatoid arthritis and systemic lupus erythematosus. Although the mechanisms behind the immunomodulatory properties of CQ and HCQ are not fully understood it is believed that they exert direct molecular effects on lysosomal activity, autophagy and immunologic signaling pathways [21, 51, 58 and 78].

CQ and HCQ are both weak bases, permitting their accumulation in acidic intracellular compartments, such as the lysosomes. Lysosomes are digestive structures and together with other vesicles like autophagosomes, recycles intracellular material and debris (autophagy) and helps processing phagocyted external antigens to be presented via MHC class II receptors to $\mathrm{T} \mathrm{CD}^{+}$lymphocytes. Autophagy and autoantigen presentation by antigen-presenting cells to $\mathrm{CD}^{+} \mathrm{T}$ cells is a probable mechanism behind the onset of some autoimmune diseases. CQ can destabilize lysosomal membranes and thus provoke the leakage of lysosomal enzymes into the cytoplasm. Furthermore, the lysosomal $\mathrm{pH}$ is optimal for the hydrolytic enzymes' activity. By increasing the $\mathrm{pH}$ inside the lysosomes, CQ and HCQ might impair the maturation of the vesicle and inhibit antigen presentation and subsequent immune activation (Figure 1) [58]. It is also proposed that CQ and HCQ inhibits autophagy mainly by provoking a severe disorganization of the Golgi and endo- lysosomal systems and therefore impairing autophagosome fusion with lysosomes [44].

CQ and HCQ also interfere with the toll-like receptor (TLR) signaling pathway. TLR are membrane spanning receptors that recognize structurally conserved molecules derived from pathogens (such as viral nucleic acids) that are usually expressed on sentinel cells like macrophages and dendritic cells. TLR can also be found in the endoplasmic reticulum of these cells. Upon ligand binding or phagocytosis, TLR can either be internalized or transported from the endoplasmic reticulum to endolysosomes where they are cleaved by lysosomal enzymes and thus becoming active. Once activated TLR triggers a signaling cascade that leads to the expression of proinflammatory cytokines such as, IL-1, IL-6, IFN-g and TNF-a. CQ and $\mathrm{HCQ}$, change the endosomal $\mathrm{pH}$, interfering in enzymatic TLR activation. Both drugs can also directly bind to the pathogen nucleic acid inside the endosome, and hence steric blocking the linkage of TLR to its ligand molecule [21, 57].

\subsection{Antiviral Effects}

As weak bases, CQ and HCQ are only capable of crossing membranes whenever in the non-protonated form. When entering acidic organelles such as the endosome, Golgi vesicles, and lysosomes these molecules become protonated and thus imprisoned and accumulate. Some viruses, such as coronaviruses, enter their host cells by endocytosis, targeting the virus to lysosomal vesicles where the low $\mathrm{pH}$ and hydrolases will disrupt the viral particle, hence liberating its nucleic acid. Accumulated CQ and HCQ in the lysosomes raise the local $\mathrm{pH}$ impairing this $\mathrm{pH}$-dependent step for viral entry. CQ can also interfere with the post-translational 
modification of viral proteins which usually involve proteases and glycosyltransferases within the endoplasmic reticulum or the trans-Golgi network vesicles, where a low $\mathrm{pH}$ is required $[73,74]$. CQ also seems to interfere with the terminal glycosylation of cellular ACE-2 receptors, the receptor that SARS-CoVs target for cell entry, a probable mechanism for preventing the virus infection and spread $[8,58]$.

\section{What is being overlooked?}

\subsection{CQ and HCQ Stereopharmacology}

The parent molecule for $\mathrm{CQ}(\mathrm{C} 18 \mathrm{H} 26 \mathrm{ClN} 3)$ is quinine, and HCQ $(\mathrm{C} 18 \mathrm{H} 26 \mathrm{ClN} 3 \mathrm{O})$ is CQ's hydroxyl derivative. Both are alkylated 4aminoquinolines and amphiphilic weak bases based on two fused aromatic rings having conjugated double bonds, the 4-aminoquinoline nucleus. Both drugs are water soluble and cross cell membranes well but because HCQ is more polar and hence less lipophilic, it has more difficulty diffusing across cell membranes. CQ and HCQ have two basic groups corresponding to the quinoline-ring nitrogen and the diethylamino side-chain nitrogen that are thought to contribute to the accumulation of these drugs in intracellular compartments, especially lysosomal compartments, which seems to be paramount for their activity and potential interaction with nucleic acids. At a physiologic $\mathrm{pH}$ of 7.4, $\mathrm{CQ}$ is monoprotonated but still soluble in lipid and able to traverse cell membranes. However, biprotonated CQ will occur in acidic organelles, such as the lysosome ( $\mathrm{pH}$ of 4-5), and be sequestered and prevented from traversing back out to the cytoplasm [53].

Both CQ and HCQ have a chiral carbon linked to the secondary amine, therefore they occur as enantiomers ( $\mathrm{R}$ and $\mathrm{S}$ isomers) (Figure 4). 


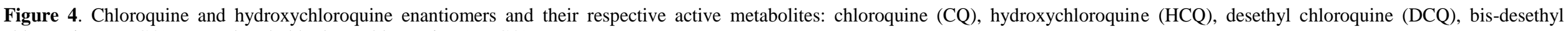
chloroquine (BDCQ), mono desethyl hydroxychloroquine (DHCQ).

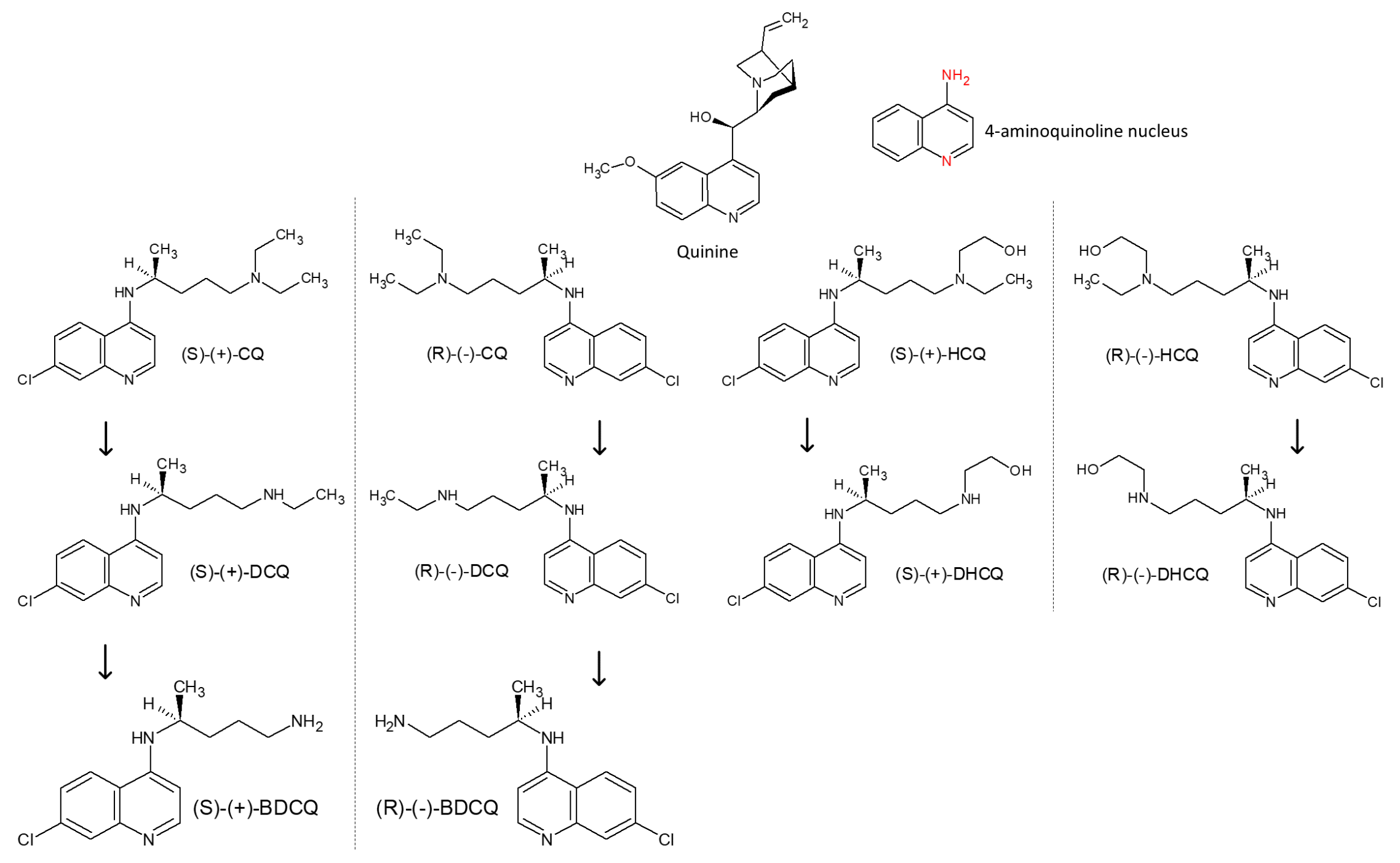


As chiral drugs, both enantiomers exhibit distinct pharmacokinetics, efficacy and toxicity profiles that can be translated into performance variances that could suggest which enantiomer can be a viable, less toxic, therapeutic agent. Eutomer is the name given to the pharmacologically active enantiomer, that is, it is the one that fits perfectly in the pharmacological receptor, causing the desired therapeutic responses. Distomer is the name given to the enantiomer that does not fit or does not fit perfectly with the pharmacological receptor. In general, the dystomer is responsible for toxic and undesirable side effects $[2,53]$. Although the interconversion between enantiomers might occur in vivo for some drugs, CQ and HCQ are stereochemically stable and the interconversion between the two enantiomers has not been observed [8, 24, 38 and 58].

Regarding pharmacokinetics, CQ and HCQ are almost completely absorbed orally within 2 to 4 hours after administration, with a plasma peak in about 3 to 12 hours. Due to their ability to be sequestered inside tissues, CQ and HCQ have a high volume of distribution and long halflives estimated at 40 - 60 days. About 33 to $70 \%$ of the bioavailable dose of CQ binds to plasma proteins, being S-CQ more highly bound to plasma proteins than R-CQ. CQ and HCQ are metabolized by the liver undergoing dealkylation. Its main metabolites are the desethyl and bisdesethyl forms, all pharmacologically active. The main route of elimination is renal, with 40 to $60 \%$ being excreted unchanged or metabolized. The S enantiomer for both CQ and HCQ is excreted by the kidneys preferentially compared to the R enantiomer [8, 14 and 37] meaning that $\mathrm{R}-\mathrm{CQ}$ is more bioavailable than its $\mathrm{S}$ counterpart.

Regarding efficacy and toxic features, CQ and HCQ binds strongly to melanin-containing tissues like the skin and the eyes, which might explain certain tissue-specific mechanisms, such as retinopathy, or the efficacy in the treatment of skin manifestations. Since sequestration of the R-CQ enantiomer in ocular tissues is greater than that of the S-CQ enantiomer, racemates are more prone to retinopathy. Furthermore, CQ has proarrhythmic activity. It prolongs the QT interval of the electrocardiogram and causes the potentially lethal long QT syndrome. This is due to its capacity to inhibit the cardiac inward rectifier potassium current, which can trigger lethal ventricular arrhythmias. Studies revealed that CQ selectively blocks cardiac potassium ion channels in the cytoplasmic portion through a three-point interaction, indicating that the three-dimensional fit may be stereo specific. Therefore, it is possible that one of CQ enantiomers might display weaker interactions with respect to its optically active counterpart, thus resulting in a lesser detrimental effect on cardiac function $[7,11]$.

It has been demonstrated that S-CQ has a higher antimalarial activity than R-CQ [38]. Results from in vitro antiviral activity against SARSCoV-2 in Vero E6 cells showed that both CQ and HCQ enantiomers exhibited antiviral effect in a concentration-dependent manner. The antiviral activity of S-CQ was relatively similar to the racemic CQ, whereas the efficacy of the S-HCQ was much better than its racemates [50]. There is still no data on the role of stereochemistry in the antiinflammatory effects of CQ and HCQ. However, the available data suggest that S-HCQ act as the eutomer and therefore it may be a more effective and less toxic alternative for the treatment of malaria, autoimmune inflammatory diseases and even COVID-19.

\subsection{Switch or not switch: what are the advantages?}

About half of the drugs currently available on the market are chiral drugs, that is, they have at least one chiral center and, therefore, are presented in at least two stereoisomeric forms as a pair of enantiomers. Among chiral drugs, approximately $90 \%$ are racemates [43, 50 and 63].

The relevance of chirality in the pharmaceutical industry became evident from the famous case of thalidomide in the 1960s. Thalidomide was sold as a racemate and used to be prescribed to ameliorate the nausea commonly felt during the first trimester of pregnancy. Unfortunately, it was discovered later that the S-enantiomer of thalidomide is teratogenic, causing the malformation of thousands of born babies. From this discovery, the Food and Drug Administration
(FDA), started to demand a more rigorous evaluation of chiral drugs [48]. CQ was familiar to the Germans as early as 1934 but was industrially developed because of intense antimalarial drug development efforts in the USA during World War II. The relative safety, efficacy, and low cost brought CQ to the front lines to treat malaria, and it was extensively used for almost two decades after its first introduction in 1944-45 [1,9 and 63]. According to today's regulations, CQ probably would not had been approved as a racemate. This ended up triggering the study and development of new models for safer, more effective and with a better therapeutic profile chiral drug. Therefore, one strategy adopted by the pharmaceutical industry for safer drugs development is chiral switching, which is the replacement of a racemate approved on the market by one of its pure enantiomers.

Chiral switching is not new to the pharmaceutical industry. Since the mid-1990s, this practice has become common as an alternative to the extension of patent protection against the production of generic racemate drugs. Successful examples are the drugs Lipitor (atorvastatin), Plavix (clopidogrel bisulfate) and Nexium (esomeprazole), which were the globally best-selling drugs in 2008 and together generated 30 billion dollars for their manufacturing laboratories. The active pharmaceutical ingredients of these medicines are the pure enantiomers that have been registered and protected under patents that derived from the patents of their corresponding racemates $[2,63]$.

From a clinical point of view, the main advantage of chiral switching is the removal of the distomer from the formulation. As a result, there are improvements in the therapeutic index, lower adverse effects, and the possibility of higher dose levels and/or longer periods of administration, besides facilitating safety and efficacy studies [37].

The chiral switch of HCQ was first accomplished in the early 1990s claiming the $\mathrm{S}$ enantiomer for treatments of malaria, rheumatoid arthritis, and lupus erythematosus. Later studies on HCQ and CQ enantiomers have been conducted but none have led to a singleenantiomer drug approval for any indication. Nevertheless, several syntheses of HCQ and CQ enantiomers have been reported, including a simple method for large-scale production of the CQ enantiomers. In the current pandemic context, emergency drug approvals should be considered. The toxicity profiles of CQ and HCQ have been well known for many years. This could speed up the approval process and a successful chiral switch of HCQ or CQ could become highly advantageous [37].

Furthermore, considering that in the formulation of a drug the substance of therapeutic interest is the eutomer, it can be concluded that in a racemate there are $50 \%$ of impurities that, in addition to not bringing any benefit to the patient, may still offer risk. Therefore, high enantiomeric purity values are mandatory for single enantiomers drugs. In this case, the distomers are impurities and must be controlled and monitored in the finished products. To do so, fast and convenient methods using high performance liquid chromatography and chiral stationary phases for assessing enantiomeric purity values of $\mathrm{CQ}$ enantiomers are now available [11, 13 and 35].

Finally, the commercialization of the pure CQ and HCQ enantiomers can be a way to prevent counterfeiting of the drug, and the evaluation of the enantiomeric purity can be a quick way to identify and rule out false or substandard drugs allegedly marketed as pure enantiomers.

\section{Discussion and conclusions}

Although new vaccines are being developed and put to the test in record time, the immune dynamics of SARS-CoV-2 imposes major challenges to be overcome. The rapid decline of $\mathrm{IgG}$ in exposed patients and the risk of antibody-dependent enhancement (ADE) are challenges that can compromise the safety of new vaccines under development. Without a lasting immune response, the risk of reinfection exists, and it is possible that COVID-19 will reappear in recurring cycles. Safe and efficient drugs that can be used for prophylaxis and prevention of the severe 
form of this disease are highly desirable. Although recent studies have also demonstrated the ineffectiveness of QC and HCQ in the treatment of mild hospitalized cases of COVID-19 [4]. Studies to evaluate the efficacy of these drugs in outpatients and in the prophylaxis of individuals frequently exposed, such as health professionals, continue. CQ and HCQ are drugs with great prophylactic and treatment potential, but their toxic side effects must be considered to prevent them from being indiscriminately used for this purpose, as a single dose of 30 $\mathrm{mg} / \mathrm{kg}$ can be fatal [26]. CQ and HCQ have already shown to be effective in vitro in reducing the infection of other viruses, such as the dengue, zika, influenza and SARS viruses. Apparently, the antiviral action of CQ and HCQ occurs by inhibiting the endosomal viral replication pathway that is common to many enveloped viruses. This mechanism of action seems to be related only to the physicochemical properties of the molecules. Although the work of Li et al (2020) has reported that the S-HCQ is more effective against SARS-CoV-2 than the racemate or its $\mathrm{R}$ counterpart, there is no further explanation about the mechanism behind this stereoselectivity. Nevertheless, while scientists are in a hurry trying to find a safe and effective dose for the prophylactic use of HCQ, Li's work prospects that the use of S-HCQ enantiomerically pure might be a way to eliminate the adverse toxic effects already attributed to the $\mathrm{R}$ form, probably allowing to double the therapeutic index of HCQ and a wider dose-response range for evaluation of the effectiveness against COVID-19.

Since CQ and HCQ are currently marketed as racemates, chiral switching these drugs might also benefit rheumatoid arthritis and lupus erythematosus patients to whom CQ and HCQ are well established as part of the treatment protocol. The adoption of the eutomer alone could allow the reduction of the administered dose with the maintenance of the same therapeutic effect, and reduction in toxicity and metabolic burden, all of which are highly desirable features for the chronic use of drugs.

In addition to potential clinical benefits, the adoption of CQ and HCQ eutomers as the only marketed molecule could be commercially advantageous and a useful asset against counterfeiting. CQ has a long history of counterfeiting and substandard commercialization especially in regions where malaria still is endemic [5]. The increase in demand and shortage of CQ and HCQ in the market due to COVID-19, besides raising the prices imposes a risk to treatment continuity of autoimmune disease patients. This has brought a golden opportunity for counterfeiting and commercialization of substandard CQ and HCQ medicines, which has raised worldwide. Simple methods for large-scale production of CQ enantiomers are promptly available. Chiral switching these drugs would discourage counterfeiting since CQ and HCQ distomers would become impurities. Convenient and quick evaluation methods, such as the determination of enantiomeric purity, could then become a useful tool in the identification of counterfeited and substandard formulations.

\section{References}

1. Abu-Raya, B., Gantt, S., \& Sadarangani, M. (2020) Challenges in evaluating SARS-CoV-2 vaccines during the COVID-19 pandemic. CMAJ : Canadian Medical Association Journal = Journal de l'Association Medicale Canadienne.

2. Agranat, I. (2020) Chiral switches of chloroquine and hydroxychloroquine: potential drugs to treat COVID-19. Drug Discovery Today, 19-21.

3. Agranat, I., \& Wainschtein, S. R. (2010) The strategy of enantiomer patents of drugs. Drug Discovery Today, 15(5-6), 163-170.

4. Al-Kofahi, M., Jacobson, P., Boulware, D. R., Matas, A., Kandaswamy, R., et al. (2020) Finding the Dose for Hydroxychloroquine Prophylaxis for COVID-19: The Desperate Search for Effectiveness. Clinical Pharmacology \& Therapeutics, cpt.1874.

5. Alves Monteiro, M., Santana Novotný, T., \& de Mendonça Ochs, S. (2020) Vigilância Sanitária de Produtos e Falsificações no Combate à Covid-19: Cloroquina e Demais Produtos Sanitary Surveillance of Medicines and Counterfeit in Combating Covid-19: Chloroquine and other products. Brazilian Journal of Health Review Braz. J. Hea. Rev, 3(4), 8357-8370.

6. Antibody levels in recovered COVID-19 patients decline quickly: research - Reuters. (n.d.). Retrieved July 17, 2020.

7. Brocks, D. R., \& Mehvar, R. (2003) Stereoselectivity in the Pharmacodynamics and Pharmacokinetics of the Chiral Antimalarial Drugs. Clinical Pharmacokinetics, 42(15), 1359 1382.

8. Browning, D. J., \& Browning, D. J. (2014) Pharmacology of Chloroquine and Hydroxychloroquine. In Hydroxychloroquine and Chloroquine Retinopathy (pp. 35-63). Springer New York.

9. Calcaterra, A., \& D'Acquarica, I. (2018) The market of chiral drugs: Chiral switches versus de novo enantiomerically pure compounds. Journal of Pharmaceutical and Biomedical Analysis, 147, 323-340.

10. Cardoso, C. D., \& Bonato, P. S. (2009) Enantioselective metabolism of hydroxychloroquine employing rats and mice hepatic microsomes. Brazilian Journal of Pharmaceutical Sciences, 45(4), 659-667.

11. Cardoso, P. A., Pereira, D. B., El-Behairy, M. F., Ghanem, A., Pianetti, G. A., \& César, I. C. (2019) Chirobiotic v versus chiralpak ID for the enantioselective chromatographic separation of chloroquine: Stability and validation study. Journal of Chromatographic Science, 57(5), 443-450.

12. Cascella, M., Rajnik, M., Cuomo, A., Dulebohn, S. C., \& di Napoli, R. (2020) Features, Evaluation and Treatment Coronavirus (COVID-19). In StatPearls. StatPearls Publishing.

13. Cavalcanti, A. B., Zampieri, F. G., Rosa, R. G., Azevedo, L. C. P., Veiga, V. C., et al. (2020) Hydroxychloroquine with or without Azithromycin in Mild-to-Moderate Covid-19. New England Journal of Medicine, NEJMoa2019014.

14. Chatre, C., Roubille, F., Vernhet, H., Jorgensen, C., \& Pers, Y.M. (2018) Cardiac Complications Attributed to Chloroquine and Hydroxychloroquine: A Systematic Review of the Literature. Drug Safety, 41(10), 919-931.

15. Chen, G., Wu, D., Guo, W., Cao, Y., Huang, D., et al. (2020) Clinical and immunological features of severe and moderate coronavirus disease 2019. Journal of Clinical Investigation, 130(5), 2620-2629.

16. Colson, P., Rolain, J. M., \& Raoult, D. (2020) Chloroquine for the 2019 novel coronavirus SARS-CoV-2. In International Journal of Antimicrobial Agents (Vol. 55, Issue 3, p. 105923). Elsevier B.V.

17. Coronavirus and hydroxychloroquine: What do we know? BBC News. (n.d.). Retrieved July 5, 2020.

18. Coronavirus disease (COVID-19). (n.d.). Retrieved January 7 , 2021.

19. Coronavirus fuels a surge in fake medicines - BBC News. (n.d.). Retrieved July 10, 2020.

20. COVID-19 vaccines. (n.d.). Retrieved January 7, 2021.

21. Devaux, C. A., Rolain, J. M., Colson, P., \& Raoult, D. (2020) New insights on the antiviral effects of chloroquine against coronavirus: what to expect for COVID-19? International Journal of Antimicrobial Agents, 55(December 2019).

22. Donoghue, M., Hsieh, F., Baronas, E., Godbout, K., Gosselin, M., et al. (2000) UltraRapid Communication A Novel Angiotensin-Converting Enzyme - Related to Angiotensin 1-9. Circ Res, 87, e1-e9.

23. Duan, G. (2020) of COVID-19. Virology, Epidemiology, Pathogenesis, and Control of COVID-19, 1-17.

24. Ducharme, J., \& Farinotti, R. (1996) Clinical pharmacokinetics and metabolism of chloroquine. Focus on recent advancements. Clinical Pharmacokinetics, 31(4), 257-274.

25. Favalli, E. G., Ingegnoli, F., de Lucia, O., Cincinelli, G., Cimaz, R., \& Caporali, R. (2020) COVID-19 infection and rheumatoid 
arthritis: Faraway, so close! Autoimmunity Reviews, 19(5), 102523.

26. FIOCRUZ. (2020) Orientações sobre o uso da Cloroquina para tratamento de pacientes infectados com SARS-CoV-2, agente etiológico da Covid-19.

27. Fu, Y., Cheng, Y., \& Wu, Y. (2020) Understanding SARSCoV-2-Mediated Inflammatory Responses: From Mechanisms to Potential Therapeutic Tools. Virologica Sinica, 12250.

28. Gautret, P., Lagier, J.-C., Parola, P., Hoang, V. T., Meddeb, L., et al. (2020) Hydroxychloroquine and azithromycin as a treatment of COVID-19: results of an open-label nonrandomized clinical trial. International Journal of Antimicrobial Agents, 105949.

29. Gellad, W. F., Choi, P., Mizah, M., Good, C. B., \& Kesselheim, A. S. (2014) Assessing the chiral switch: Approval and use of single-enantiomer drugs, 2001 to 2011. American Journal of Managed Care, 20(3).

30. Guan, G. W., Gao, L., Wang, J. W., Wen, X. J., Mao, Tet al. (2020) Exploring the mechanism of liver enzyme abnormalities in patients with novel coronavirus-infected pneumonia. Zhonghua Gan Zang Bing Za Zhi = Zhonghua Ganzangbing Zazhi $=$ Chinese Journal of Hepatology, 28(2), 100-106.

31. He, F., Deng, Y., \& Li, W. (2020) Coronavirus disease 2019: What we know? Journal of Medical Virology, March, 1-7.

32. Hoffmann, M., Kleine-Weber, H., \& Pöhlmann, S. (2020) A Multibasic Cleavage Site in the Spike Protein of SARS-CoV-2 Is Essential for Infection of Human Lung Cells. Molecular Cell, 78(4), 779-784.e5.

33. Hoffmann, M., Kleine-Weber, H., Schroeder, S., Krüger, N., Herrler, T., et al. (2020) SARS-CoV-2 Cell Entry Depends on ACE2 and TMPRSS2 and Is Blocked by a Clinically Proven Protease Inhibitor. Cell, 181(2), 271-280.e8.

34. Home - ClinicalTrials.gov. (n.d.). Retrieved July 5, 2020.

35. Ibrahim, K. E., \& Fell, A. F. (1990) Separation of chloroquine enantiomers by high-performance liquid chromatography. Journal of Pharmaceutical and Biomedical Analysis, 8(5), 449452.

36. Imai, Y., Kuba, K., \& Penninger, J. M. (2008) The discovery of angiotensin-converting enzyme 2 and its role in acute lung injury in mice. Experimental Physiology, 93(5), 543-548.

37. Lentini, G., Cavalluzzi, M. M., \& Habtemariam, S. (2020) Safety Concern : Chirality Might Help. Molecules, 25(Figure 1), 1834.

38. Li, G., Sun, J., Li, Y., Shi, Y., Zhao, J., Zhang, T. Y., \& Zhang, X. (2020) Enantiomers of Chloroquine and Hydroxychloroquine Exhibit Different Activities Against SARS-CoV-2 in vitro, Evidencing S-Hydroxychloroquine as a Potentially Superior Drug for COVID-19. BioRxiv, 2020.05.26.114033.

39. Li, X. C., Zhang, J., \& Zhuo, J. L. (2017) The vasoprotective axes of the renin-angiotensin system: Physiological relevance and therapeutic implications in cardiovascular, hypertensive and kidney diseases. Pharmacological Research, 125, 21-38.

40. Liu, C., Zhou, Q., Li, Y., Garner, L. v., Watkins, S. P., et al. (2020) Research and Development on Therapeutic Agents and Vaccines for COVID-19 and Related Human Coronavirus Diseases. ACS Central Science, 6(3), 315-331.

41. Liu, J., Cao, R., Xu, M., Wang, X., Zhang, H., et al. (2020) Hydroxychloroquine, a less toxic derivative of chloroquine, is effective in inhibiting SARS-CoV-2 infection in vitro. In Cell Discovery (Vol. 6, Issue 1). Springer Nature.

42. Long, Q. X., Tang, X. J., Shi, Q. L., Li, Q., Deng, H. J., et al. (2020) Clinical and immunological assessment of asymptomatic SARS-CoV-2 infections. Nature Medicine, 1-5.

43. Mane, S. (2016) Racemic drug resolution: A comprehensive guide. Analytical Methods, 8(42), 7567-7586.

44. Mauthe, M., Orhon, I., Rocchi, C., Zhou, X., Luhr, M., et al. (2018). Chloroquine inhibits autophagic flux by decreasing autophagosome-lysosome fusion. Autophagy, 14(8), 14351455.

45. Medical Product Alert $N^{\circ} 4 / 2020$. (n.d.). Retrieved July 10, 2020.

46. Mehra, M. R., Desai, S. S., Ruschitzka, F., \& Patel, A. N. (2020) Hydroxychloroquine or chloroquine with or without a macrolide for treatment of COVID-19: a multinational registry analysis. Lancet (London, England), 6736(20), 1-10.

47. Monteiro, W. M., Brito-Sousa, J. D., Baía-Da-Silva, D., de Melo, G. C., Siqueira, A. M., Val, F., Daniel-Ribeiro, C. T., \& Lacerda, M. V. G. (2020) Driving forces for covid-19 clinical trials using chloroquine: The need to choose the right research questions and outcomes. In Revista da Sociedade Brasileira de Medicina Tropical (Vol. 53). Sociedade Brasileira de Medicina Tropical.

48. Muraleedharan, K. M., \& Avery, M. A. (2006) Advances in the discovery of new antimalarials. Comprehensive Medicinal Chemistry II, 7, 765-814.

49. Negro, F. (2020) Is antibody-dependent enhancement playing a role in COVID-19 pathogenesis? Swiss Medical Weekly, 150(April), w20249.

50. Nguyen, L. A., He, H., \& Pham-Huy, C. (2006) Chiral drugs: an overview. International Journal of Biomedical Science: IJBS, 2(2), 85-100.

51. Oh, S., Shin, J. H., Jang, E. J., Won, H. Y., Kim, H. K., Jeong, M. G., Kim, K. S., \& Hwang, E. S. (2016) Anti-inflammatory activity of chloroquine and amodiaquine through p21-mediated suppression of $\mathrm{T}$ cell proliferation and Th1 cell differentiation. Biochemical and Biophysical Research Communications, 474(2), 345-350.

52. Patel, V. B., Parajuli, N., \& Oudit, G. Y. (2014) Role of angiotensin-converting enzyme 2 (ACE2) in diabetic cardiovascular complications. Clinical Science, 126(7), 471482.

53. Patocka, J., \& Dworak, A. (2004) Biomedical aspects of chiral molecules. Journal of Applied Biomedicine, 2, 95-100.

54. Paumgartten, F. J. R., Delgado, I. F., Pitta, L. D. R., \& Oliveira, A. C. A. X. de. (2020) Drug repurposing clinical trials in the search for life-saving COVID-19 therapies; research targets and methodological and ethical issues. Vigilância Sanitária Em Debate, 8(2), 39-53.

55. Pedersen, S. F., \& Ho, Y. C. (2020) SARS-CoV-2: A storm is raging. Journal of Clinical Investigation, 130(5), 2202-2205.

56. Ria, V., Palmeira, A., Braga Costa, L., Giandoni Perez, L., Ribeiro, V. T., Lanza, K., \& Cristina, A. (2020) Do we have enough evidence to use chloroquine/ hydroxychloroquine as a public health panacea for COVID-19? Clinics, 75.

57. Savarino, A., Boelaert, J. R., Cassone, A., Majori, G., \& Cauda, R. (2003) Effects of chloroquine on viral infections: An old drug against today's diseases? Lancet Infectious Diseases, 3(11), 722-727.

58. Schrezenmeier, E., \& Dörner, T. (2020) Mechanisms of action of hydroxychloroquine and chloroquine: implications for rheumatology. Nature Reviews Rheumatology, 16(3), 155-166.

59. Singh, A. K., Gupta, R., Ghosh, A., \& Misra, A. (2020) Diabetes in COVID-19: Prevalence, pathophysiology, prognosis and practical considerations. Diabetes and Metabolic Syndrome: Clinical Research and Reviews, 14(4), 303-310.

60. Skipper, C. P., Pastick, K. A., Engen, N. W., Bangdiwala, A. S., Abassi, M., et al. (2020) Hydroxychloroquine in Nonhospitalized Adults With Early COVID-19. Annals of Internal Medicine, M20-4207.

61. Solidarity clinical trial for COVID-19 treatments . (n.d.) Retrieved July 10, 2020.

62. Stock, P., Liefeldt, L., Paul, M., \& Ganten, D. (1995) Local renin-angiotensin systems in cardiovascular tissues: 
Localization and functional role. Cardiology (Switzerland), 86, $2-8$.

63. Sumithira, G., \& Sujatha, M. (2013) Drug chirality \& its clinical significance evident, future for the development/separation of single enantiomer drug from racemates - The chiral switch. International Journal of Advanced Pharmaceutical Research, $1(1), 1-19$.

64. Swank, K., \& Mccartan, K. (2020) Application Type/Number: NDA 009768, ANDA multiple.

65. Tetro, J. A. (2020) Is COVID-19 receiving ADE from other coronaviruses? Microbes and Infection, 22(2), 72-73.

66. Timeline of WHO's response to COVID-19. (n.d.). Retrieved June 28, 2020.

67. Trade, F., For, S., Economic, I., Financial, I., \& Unit, M. (2020) Brazil 's Policy Responses to COVID-19. 2020-2023.

68. True claim: Brazilian chloroquine study on COVID-19 patients was halted after 11 deaths - Reuters. (n.d.). Retrieved July 10, 2020.

69. Tucker, G. T. (2000) New drug classes: Chiral switches. The Lancet, 355, 1085-1087.

70. Vellingiri, B., Jayaramayya, K., Iyer, M., Narayanasamy, A., Govindasamy, V., et al. (2020) COVID-19: A promising cure for the global panic. Science of the Total Environment, 725, 138277.

71. Vincent, M. J., Bergeron, E., Benjannet, S., Erickson, B. R., Rollin, P. E., et al. (2005) Chloroquine is a potent inhibitor of SARS coronavirus infection and spread. Virology Journal, 2, 110.

72. Wan, Y., Shang, J., Sun, S., Tai, W., Chen, J., et al. (2019) Molecular Mechanism for Antibody-Dependent Enhancement of Coronavirus Entry. Journal of Virology, 94(5), 2015-2034.

73. Wang, M., Cao, R., Zhang, L., Yang, X., Liu, J., et al. (2020) Remdesivir and chloroquine effectively inhibit the recently emerged novel coronavirus $(2019-\mathrm{nCoV})$ in vitro. Cell Research, 30(3), 269-271.

74. Wang, X., Khaidakov, M., Ding, Z., Mitra, S., Lu, J., Liu, S., \& Mehta, J. L. (2012) Cross-talk between inflammation and angiotensin II: Studies based on direct transfection of cardiomyocytes with AT1R and AT2R cDNA. Experimental Biology and Medicine, 237(12), 1394-1401.

75. Why we might not get a coronavirus vaccine | Coronavirus outbreak | The Guardian. (n.d.). Retrieved June 28, 2020.

76. Yang, M. (2020) Cell Pyroptosis, a Potential Pathogenic Mechanism of 2019-nCoV Infection. SSRN Electronic Journal.

77. Ye, Q., Wang, B., \& Mao, J. (2020) The pathogenesis and treatment of the 'Cytokine Storm' in COVID-19.' Journal of Infection, 80(6), 607-613.

78. Zhao, M. (2020) Cytokine storm and immunomodulatory therapy in COVID-19: Role of chloroquine and anti-IL-6 monoclonal antibodies. International Journal of Antimicrobial Agents, 55(6), 105982. 